\title{
Sur la rétention des traces de fission de l'uranium dans les minéraux
}

\author{
D. Chaillou, A. Chambaudet \\ Laboratoire de Chimie Physique (*), avenue Jean-Perrin, Bâtiment 350, 91405 Campus d’Orsay, France
}

et B. Zidler

Processus Photophysiques et Photochimiques (**), Bâtiment 350, Campus d'Orsay, France

(Reçu le 19 décembre 1980, révisé le 8 avril 1981, accepté le 2 juin 1981)

\begin{abstract}
Résumé. - L'étude sur la stabilité thermique des traces latentes de fission de l'uranium enregistrées dans des apatites (Massif Armoricain, Bretagne), nous a conduit à proposer une nouvelle fonction mathématique $\left(\bar{\rho}(t)=\sum_{i} \bar{\rho}_{0 i} \exp \left(-\alpha_{i} t\right)\right)$ beaucoup mieux adaptée que celles avancées jusqu'à maintenant pour représenter la cinétique de réduction des traces de fission au cours d'un recuit isotherme. Ce résultat est par ailleurs en très bon accord avec la théorie avancée récemment $[1,2]$ sur la structure des traces latentes enregistrées dans les minéraux (muscovite, olivine et labradorite).

Abstract. - We propose a new mathematical function $\left(\bar{\rho}(t)=\sum_{i} \bar{\rho}_{0 i} \exp \left(-\alpha_{i} t\right)\right)$ to describe the kinetics of uranium fission track reduction during an isothermal annealing process based on a study of the stability of latent uranium fission tracks in apatite samples from the hercynian platform in French Brittany. This result is in very good agreement with a recent theory $[1,2]$ on the structure of latent tracks in minerals (muscovite, olivine and labradorite).
\end{abstract}

1. Introduction. - Les premières datations par la méthode des traces de fission datent de 1963 [3]. Le principe en est simple : dans une roche naturelle contenant de l'uranium, la fission spontanée d'un atome père produit deux noyaux fils qui, en se ralentissant de part et d'autre de celui-ci, laissent une zone de dégâts (trace latente); un traitement chimique approprié rend cette zone observable par microscopie optique ou électronique (trace révélée). Parmi les isotopes naturels qui fissionnent spontanément ${ }^{232} \mathrm{Th}$, ${ }^{235} \mathrm{U}$ et ${ }^{238} \mathrm{U}$, seul ce dernier fissionne avec une période suffisamment courte pour donner un nombre non négligeable de traces. Leur densité permet alors de déterminer l'âge du minéral si l'on connaît sa concentration en uranium. Celle-ci est obtenue en irradiant le cristal à l'aide d'un flux connu de neutrons thermiques induisant la fission des noyaux de ${ }^{235} \mathrm{U}$.
2. Stabilité des traces. - La détermination d'un âge par la méthode des traces suppose que ces dégâts soient stables. Or, comme les autres géochronomètres nucléaires, les traces de fission de l'uranium sont sensibles aux conditions d'environnement et tout particulièrement aux effets thermiques.

De tels effets ont pour conséquence de réduire la longueur des traces fossiles, conduisant ainsi à un «âge apparent » plus jeune.

Il convient donc pour corriger les «âges thermiquement rajeunis » de ramener les traces fossiles et induites dans le même état de réduction. Ceci peut être obtenu en laboratoire par un traitement thermique soit isochrone [4], soit isotherme [5].

Dans le présent article, nous nous sommes intéressés au problème concernant la cinétique "globale » de recuit en régime isotherme des traces de fission. En effet, le modèle simple adopté par la plupart des géochronologues pour évaluer les conditions de rétention des traces de fission à des temps géologiques repose sur des expérimentations en laboratoire qui sont sujettes à controverses. 
3. Choix de la température pour le recuit isotherme. - Le degré de réduction des longueurs de traces pour une durée de recuit donnée est fonction de la température ; par conséquent, il est nécessaire d'opérer à une température pas trop élevée pour pouvoir étudier la totalité du phénomène.

Le choix de la température est déduit d'une étude en recuit isochrone [6]. La température retenue $\left(270^{\circ} \mathrm{C}\right)$ correspond au début de la réduction des densités de traces.

Il est à noter que notre méthode d'expérimentation est une variante de celle de Galazka et Burchard [5] ; nous n'opérons pas par recuits successifs des mêmes cristaux (" méthode cumulative»), mais pour chaque durée de recuit un nouveau lot de cristaux du même échantillonnage est utilisé.

4. Expression mathématique de la cinétique du recuit en régime isotherme. - Pour décrier ce processus, trois hypothèses ont été envisagées : la réduction des traces $\bar{\rho}_{t} / \bar{\rho}_{0}$ est une fonction

i) linéaire de $t(t=$ durée de recuit),

ii) exponentielle de $t$,

iii) logarithmique de $t$.

Bien que cette dernière (iii) soit en général la plus utilisée, notre étude remet en question une telle hypothèse.

En effet, notre expérimentation montre des écarts significatifs avec une telle représentation (Fig. 1). D'autre part, si le processus était décrit par une fonction logarithmique, la variation de l'énergie d'activation $E$ devrait être une fonction linéaire du pourcentage de réduction des densités de traces. Nos calculs montrent que ce n'est pas le cas et permettent par contre de mettre en évidence très nettement un

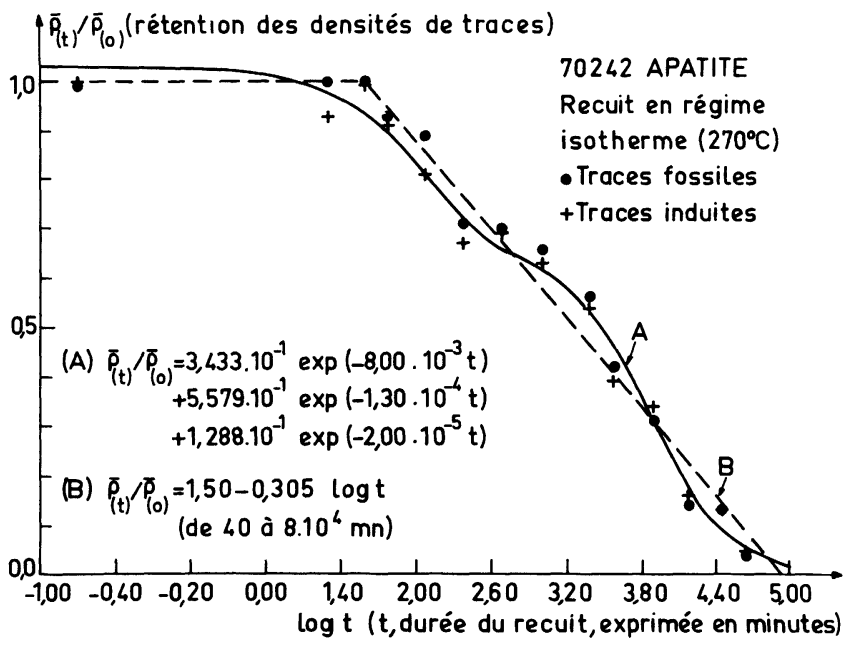

Fig. 1. - Réduction des densités de traces en fonction de la durée de recuit (la courbe en pointillé est le meilleur ajustement de la fonction logarithmique $\bar{\rho}(t)=a-b \log t$ ).

[Track density versus annealing time (the dashed line is the best fit of the logarithmic function $\bar{\rho}(t)=a-b \log t)$.] phénomène à deux étapes suggérant ainsi une différence entre le recuit des traces fossiles et des traces induites dans le cas d'un échantillon affecté thermiquement au cours de son histoire géologique.

Ceci nous a conduit à proposer une fonction mathématique adaptée à ces résultats :

$$
\bar{\rho}(t)=\bar{\rho}_{01} \exp \left(-\alpha_{1} t\right)+\bar{\rho}_{02} \exp \left(-\alpha_{2} t\right)
$$

avec

$$
\alpha_{i}(T)=\alpha_{0} \exp \left(-E_{i} / k T\right)
$$

70242 APATITE - Recuit en régime isotherme

$\left[\bar{P}_{(t)}{ }^{P} \bar{P}_{(0)}\right.$ (rétention des densités de traces)

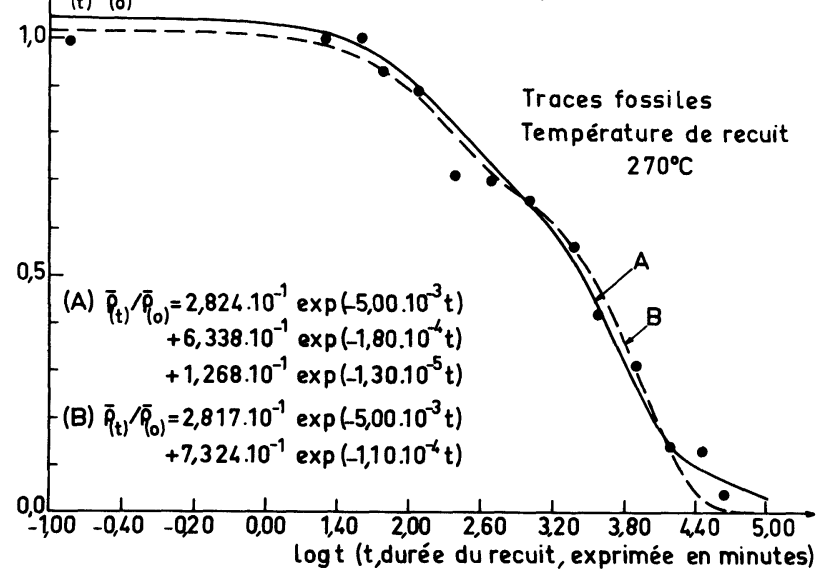

Fig. 2. - Traces fossiles : représentation des meilleurs ajustements de la fonction $\bar{\rho}(t)=\sum_{i} \bar{\rho}_{0 i} \exp \left(-\alpha_{i} t\right)$ pour $i=2(\mathrm{~B}$, fonction (1)) et $i=3$ (A, fonction (2)).

[Fossil tracks : best fit of the function $\bar{\rho}(t)=\sum_{i} \bar{\rho}_{0 i} \exp \left(-\alpha_{i} t\right)$ for $i=2(\mathrm{~B}$, function (1)) and for $i=3$ (A, function (2)). $]$

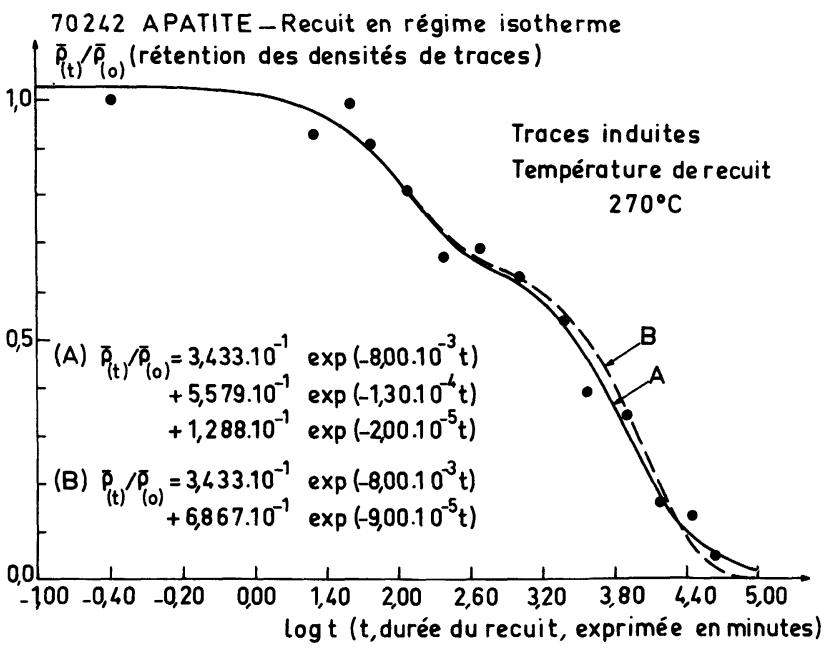

Fig. 3. - Traces induites : représentation des meilleurs ajustements de la fonction $\bar{\rho}(t)=\sum_{i} \bar{\rho}_{0 i} \exp \left(-\alpha_{i} t\right)$ pour $i=2(\mathrm{~B}$, fonction (1)) et $i=3$ (A, fonction (2)).

[Induced tracks : best fit of the function $\bar{\rho}(t)=\sum_{i} \bar{\rho}_{0 i} \exp \left(-\alpha_{i} t\right)$ for $i=2(\mathrm{~B}$, function (1)) and for $i=3(\mathrm{~A}$, function (2)). $]$ 
où $t$ est le temps de recuit,

$T$ est la température de recuit,

$E_{i}$ est l'énergie d'activation,

$k$ est la constante de Boltzmann.

L'accord d'une telle fonction avec nos résultats expérimentaux, aussi bien pour les traces fossiles (Fig. 2, B) que pour les traces induites (Fig. 3, B), est très satisfaisant et conduit à une histoire thermique simple de nos échantillons. Cependant l'introduction d'un terme supplémentaire :

$$
\begin{aligned}
\bar{\rho}(t)=\bar{\rho}_{01} \exp \left(-\alpha_{1} t\right)+\bar{\rho}_{02} & \exp \left(-\alpha_{2} t\right)+ \\
& +\bar{\rho}_{03} \exp \left(-\alpha_{3} t\right)
\end{aligned}
$$

conduit à un meilleur ajustement (Fig. 2, A ; Fig. 3, A).

5. Interprétation physique. - Nos résultats sont en bon accord avec la récente théorie de Dartyge et al. $[1,2]$ sur la structure de la trace latente dans des minéraux (muscovite, labradorite et olivine). Ces auteurs considèrent en effet qu'une trace est consti- tuée de défauts ponctuels et de défauts étendus de sensibilité différente aux effets thermiques; les défauts ponctuels "s'effacent" en premier pour une température caractéristique du minéral; les défauts étendus par contre sont étroitement corrélés à la nature de l'ion incident et "disparaissent" pour des températures supérieures.

6. Conclusion. - En conclusion, notre formulation mathématique pour décrire la cinétique de recuit en régime isotherme des traces latentes de fission de l'uranium confirme le modèle proposé par Dartyge et al. [1,2] pour rendre compte de la structure complexe des traces latentes. Cette formulation n'est pas une théorie car les différents paramètres doivent être redéterminés pour chaque température de recuit. Cependant, la fonction proposée et composée d'une somme d'exponentielles décroissantes est mieux adaptée pour représenter ce processus que toutes les fonctions mathématiques avancées jusqu'à maintenant.

\section{Bibliographie}

[1] Dartyge, E., Duraud, J. P. and Langevin, Y., Radiat. Eff. 34 (1977) 77.

[2] Dartyge, E., Duraud, J. P., Langevin, Y. and Maurette, M., Proc. 9th Lunar Sci. Conf. (1978) 2375.

[3] Pric 1., P. B. and Walker, R. M.. J.Gcophls. Res. 68 (1963) 4847.

[4] Storzer, D., J. Ber. M.P.I. Kernphlisik, Heidelberg (1972) 199.

[5] Galazka, J. and Burchard, J., Bull. Acad. Polon. Sci., Serie Sci. de la Terre 25 (1977) 1.

[6] Challlou, D., Thèse $3^{\mathrm{e}}$ cycle, Université Pierre-et-MarieCurie, Paris VI (1981). 